\title{
Iris Indexing Techniques: A Review
}

\author{
N. Poonguzhali \\ Research Scholar, Department of Computer \\ Science and Engineering \\ Pondicherry Engineering College, India
}

\author{
M. Ezhilarasan \\ Professor and Head \\ Department of Information Technology \\ Pondicherry Engineering College, India
}

\begin{abstract}
The objective of this paper is to present the state of art in iris indexing. The potential raise of accurateness along with enhanced robustness beside forgeries makes in fact iris recognition a promising field for research. The performance of a biometric system is evaluated based on the retrieval time and error rate which are dependent on the size of the database and hence the need for indexing. Iris indexing can be categorized based on the texture analysis, color and SFIT key point. Further the paper discusses the description of some databases used for indexing techniques to prove the efficiency. The performance evaluation metrics are also discussed.
\end{abstract}

\section{General Terms}

Pattern Recognition, Biometrics

\section{Keywords}

Biometric, iris, indexing, performance metrics, databases

\section{INTRODUCTION}

Traditionally, identification of a genuine person was based on key, password, magnetic or chip card. However, all of these can be stolen, forgotten or forged and hence password and token- based recognition systems are nowadays replaced by biometric recognition system. Even in some systems where password and token are still used, on top of it a biometric layer is added for more secure authentication. Hence there is a tremendous growth in biometric based identification system in almost all paths round the world. Biometric includes reference to the measurement, analysis, classification, science of personal recognition and verification or identification by using distinguishable biological (physiological) or behavioral trait, features or characteristic of that person. Biometric identification is the process of associating an identity to the input biometric data by comparing it against the enrolled identities in a database [1].

Depending on the mode of application biometric system operate on verification or identification mode. In verification mode, the system validates a person's identity by comparing the captured biometric data with their own biometric template stored in the database; say by 1:1 matching. In identification mode, the system recognizes the person by searching the template of all the users in the database, where 1: $\mathrm{N}$ matching is performed. Any biometric system should posses the characteristics such as: versatility, uniqueness, permanence, measurability, performance, acceptability and circumvention. Some of the commonly used physical or behavioural traits are: face, fingerprint, palm print, hand geometry, iris, keystroke, signature, voice and gait. Apart from these, there are also some traits which are used to identify a person uniquely based on odour, tongue, ear, knuckle print.

Among the various biometric traits, 'iris' is proved to be a predominant and popular biometric feature for authentication and identification with a high degree of accuracy and distinctiveness. The finger print has also received substantial concentration and is being effectively used in many applications over one hundred years. Face and speech have also been extensively studied over 25 years, whereas, 'iris recognition' is a newly emerging trait in personal identification and is reported to be the most reliable biometric trait in identification and authentication [2]. The performance of the identification system depends more on the search space and the accuracy rate. Existing systems are till now small-scaled, databases and henceforth identifying an imposter is not a difficult task, but, as the size grows it is necessary to identify a methodology which can search the correct person with in the stipulated time. Hence, 'indexing' becomes vital to identify the legitimate person from databases based on the templates stored.

The paper is organized as follows. The anatomy of the human iris and the feature extraction are described in section 2. An exhaustive survey on various indexing techniques based on the iris features are detailed in section 3 . Performance metric used to evaluate the techniques and description about the databases has been reviewed in section 4. A comparative study on the performance of the indexing technique in the survey is analyzed in the last section.

\section{IRIS BIOMETRIC IDENTIFICATION SYSTEM}

\subsection{Iris basics}

Iris is a Greek word means goddess of the rainbow due to many colours of the iris and hence the name. The iris is a thin circular ring region, a part of the human eye positioned between the black pupil and white sclera presenting a unique and rich texture information, such as patterns, rifts, colors, rings, spots, stripes, filaments, coronal, furrows, minutiae and recess and other details of the characteristics of the infrared light. The iris is responsible for the amount of light reaching the retina by controlling the diameter and size of the pupil which is hole a located in the center of the iris. The Iris is the only internal organ which seen from outside. Further it is measureable internal physical feature, which is unique and cannot be easily altered due to ecological effects. The probability of finding any two eyes identical, are approximately 1 in $10^{\wedge} 52$. It has nearly about 250 points of identification for comparison. The minute iris patterns and 
textures which is the phenotypic feature are determined during the foetal development and not inherited from the gene and for this reason the right and left eye of a person are not alike and same even for the identical twins. The color of the eye and structure are dictated by genotype, whereas tiny texture details are epigenetic.

Eye color is the color of the iris, which can be green, blue, or brown. In some cases it can be hazel (a combination of light brown, green and gold), grey, violet, or even pink. The idea of using iris feature for human based recognition is over hundred years old [2] but the automated recognition system is very young. Dr. Frank Burch an ophthalmologist in the year 1936 proposed iris for the purpose of identification of individuals. After a long break in 1985 Dr. Leonard Flom and Aran Safir found that no two irises are identical. Then after in 1993 Dr. John Daugman proceeded with the novel approach of Dr. Flom and Safir and effectively automated the iris recognition system by 1994 .

\subsection{Iris Feature Extraction}

The iris feature is extracted based on the method proposed by Daugman. The segmentation process of detecting the inner and outer boundaries are done using active-contour model. The success of segmentation depends on the imaging quality of eye images. The pre-segmentation process involves Gaussian smoothing to remove the noise. Mathematically the Gaussian smoothing function can be used. Daugman makes use of an integro-differential operator for locating the circular iris and pupil regions, and also the arcs of the upper and lower eyelids. In the circular path the operator travels around for a maximum changes in pixel value with respect to the radius and centre of $\mathrm{x}$ and $\mathrm{y}$ position in the circular contour. The operator is progressively used to smoothen in order attain precise localization. Eyelids are localized with the path of contour integration changed from circular to an arc. The screen shot of segmentation model is given below fig. 2 .

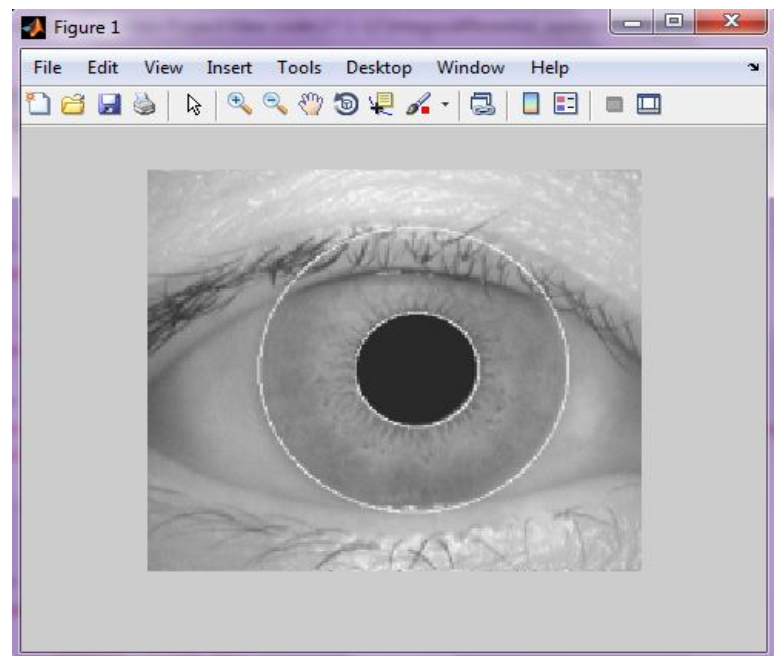

Fig. 2. Segmented Iris image

Since it works with raw derivative information, it does not suffer from the thresholding problems of the Hough transform. However, the algorithm can fail where there is noise in the eye image, such as from reflections, since it works only on a local scale.

The next process of normalization is done based on the homogenous rubber sheet model formulated by Daugman which remaps each point within the iris region to a pair of polar coordinates $(r, \theta)$ where $r$ is on the interval $[0,1]$ and $\theta$ is angle $[0,2 \pi]$ as shown in the fig. 3 .

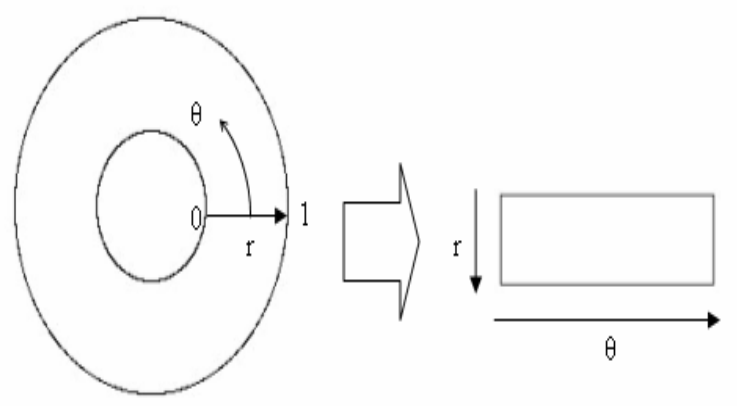

Fig.3. Daugman's Rubber Sheet Model

The rubber sheet model takes into account pupil dilation and size inconsistencies in order to produce a normalized representation with constant dimensions. In this way the iris region is modelled as a flexible rubber sheet anchored at the iris boundary with the pupil centre as the reference point. Even though the homogenous rubber sheet model accounts for pupil dilation, imaging distance and non-concentric pupil displacement, it does not compensate for rotational inconsistencies. In the Daugman's system, rotation is accounted for during matching by shifting the iris templates in the $\theta$ direction until two iris templates are aligned as shown in fig. 4.

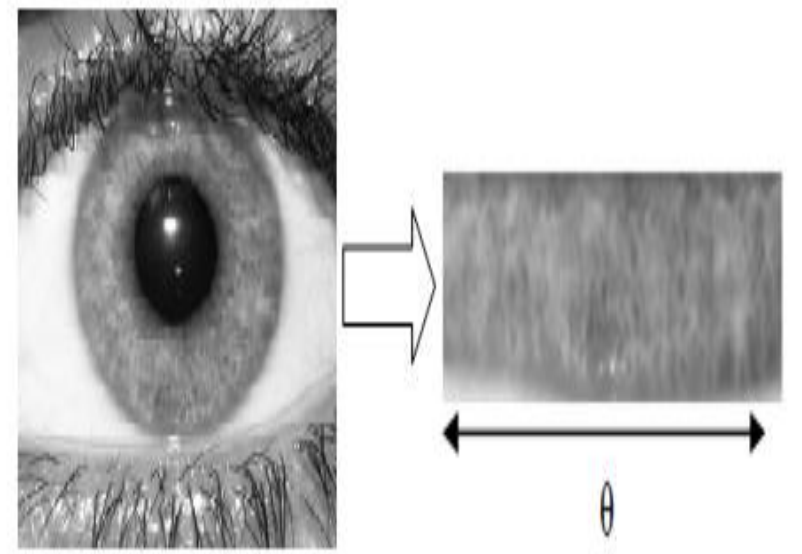

Fig. 4. Normalization of iris 
The given iris is encoded into a 256 byte value known as the Iris Code. This is done by a simple filtering operation of the iris texture using the Gabor functions as shown in fig.5.

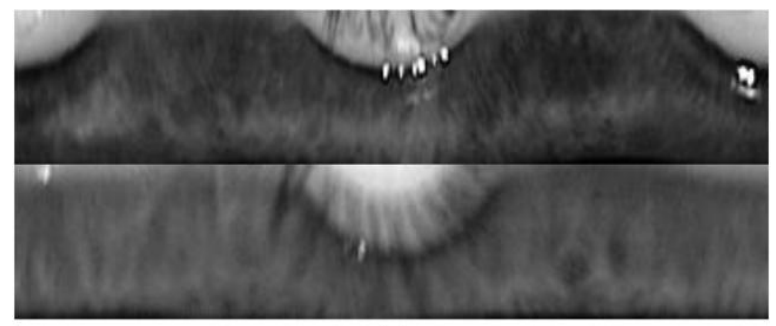

(a)

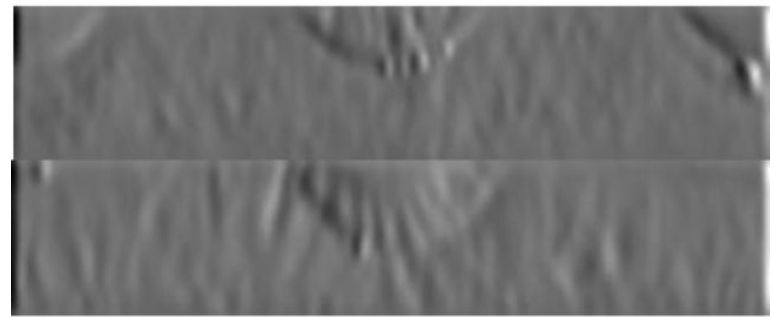

(b)

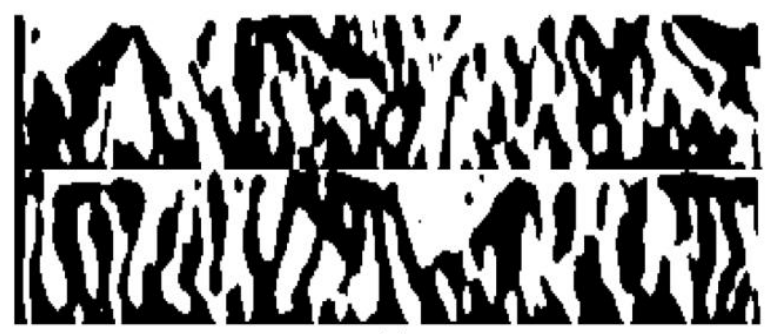

(c)

Fig. 5. a) Image obtained after normalization. b) After blurring the noise. c) iris code using Gabor filter.

Considering a single filtered response, every real and imaginary pixel value of the response is assigned a bit value of 0 or 1 based on whether the value is greater than or lesser than 0 . This way, we can encode both the real and imaginary part of the response, getting an encoded template that has two bits per pixel corresponding to four phase (quadrant) values in the complex plane. We considered only the imaginary part of the Gabor function and optimized its parameters (including a small initial phase parameter). Thus, the encoding requires only 1 bit per pixel which yields a more efficient classifier with similar performance. The final bit map for an iris in our work has a size of $60 \times 360$. The encoded versions of the filtered responses in Fig.5 (b) are shown in (c). This iris code is used as a feature vector.

\section{IRIS INDEXING TECHNIQUES}

An index is a data structure that identifies locations at which indexed values occur. In context of a database, an index identifies which records contain which value. There is no natural order by which one can sort the biometric data. Further the existing techniques work well with small sized database with respect to accuracy and time. There is an enormous increase in the size of database like a project by the Unique Identification Authority of India (UIDAI) to issue Unique Identification (UID) numbers to all residents in the country [3]. As the size grows, the computational time for performing iris identification on large databases is a challenging task and one such approach is parallel computing [4]. Parallel processing can be done in huge database where the computation time can be reduced but the cost of operation is again a challenging task. Thus for, reducing the search time and correct identification there is need for a generic indexing technique for biometric data. Iris can be classified according to its texture such as fine: fine/medium, medium/coarse, coarse and by its color: blue/grey, amber, light brown, brown, dark brown. Apart from the above, indexing technique can be classified based on iris code and SFIT key points.

\subsection{Based on Iris Code}

Iris indexing based on iris code is considered to be a pioneering work on this area proposed in [5]. In the above approach three techniques were proposed. The first is based on Principal Component Analysis (PCA) on Iriscode and kmeans algorithm is performed on to reduce the dimension vector. The second is based on the Local Binary Pattern (LBP) in which each image is divided into blocks and a histogram is calculated for all the blocks in the given image. The number of bins in the histogram depends on the radius considered to generate the LBP. The close relationship between LBP histograms is exploited to decrease the number of candidate hypothesis for matching. This technique was analyzed by varying the number of neighborhood pixels for calculating the LBP code. The third technique analyses the inter-pixel relationship of the iris. In order to extract pixel level difference features of blocks, a Signed Pixel Level Difference Histogram (SPLD) is generated. The SPLDH is computed by taking the histogram of the signed differences of the corresponding pixel positions of the two blocks. The entropy of the histogram is calculated in order to determine the importance of the second block with respect to the first block. As the work focus on comparison among the three techniques which results that, it does not perform as well as the indexing technique using block based features of IrisCode, the performance is far better than the indexing technique based on LBP. In spite of the fact that the above work is a revolutionary one for iris indexing technique the hit rate is low and penetration rate it high even for a small-scale database. However it has opened up further research on these indexing techniques.

The issue of indexing noisy data has always been challenging. In [6] one such approach is attempted. The first step is to detect the eyelash which is misclassified as texture by several algorithms. Hence a method based on the direction of the eyelash was found, which is based on the observation that every eyelash usually grows along a single direction or with a small deflection which can be observed by two directions close to each other. A connection rule is formed in order to reduce the loss as well as detect the eyelash. Based on connection rule, eye lash can be removed according to their position. The second part is feature extraction which is done based on the multiscale and multiorientation data fusion strategy after 2-D Gabor filtering which describes both the scale and direction of the iris texture features. Finally an indexing technique based on the statistical analysis of texture distribution in every block of the normalized iris image is accomplished through Harris corner detection method [7] where the unwanted corners are discarded and evenly cropped image are taken. The masked regions of the normalized iris images are marked using ternary indexing codes which guarantees both the accuracy and retrieval. The above method is compared with PCA method [5] and SIFT 
keypoint method [8], has led to the twin advantage of lower penetration rate and miss rate.

\subsection{Based on Iris Texture}

An efficient indexing scheme based on energy histogram obtained from the iris texture was proposed in [9]. Image is stored into the database along with a key during enrolment. A B-Tree data structure was used to store the key value. The acquired iris image is then pre-processed using Circular Hough transform [10] and transformed into rectangular block [11]. The transformed rectangular region is enhanced to improve the texture details and make it illumination in variant. Next, the features are extracted using multiresolution DCT (Discrete Cosine Transformation) [12]. The input image is divided into non-overlapping $8 \times 8$ pixel block based on DCT approach. The co-efficient from each block belong to a particular subband are grouped together and a feature vector value is obtained form 10 such subbands. Further, during indexing energy histogram is built for each subband. The histogram generated from each subband is divided into bin to form logical groups. The bin number is used to form key value for indexing. Insertion to the B-Tree is made at the leaf level and the key for the image is calculated by finding the bin number. The tree is traversed from the root node down to the leaf node to find the suitable match. It has been observed that scalability is a problem in this approach as the size of the bin and subband is fixed which can lead to misclassification when there is coarse texture.

This paper [13] proposed an efficient indexing technique which is used for a large multimodal biometric database. Iris was one of the traits focused in the above work and the feature vectors were normalized and projected to a lower dimensional feature vector space. The inner boundary of the iris was extracted using Circular Hough transformation [10] and the outer boundary based on intensity variation approach [14]. Then the iris feature was transformed into rectangular block and the features are extracting using Harr Wavelet Transform [15]. Later feature normalization was done and the feature vectors were used to form kd-tree. To find the similar matching the tree is traversed starting its search from the root and finds its position. A range of search result is produced and Euclidean distance is computed to identify the correct match. The above technique has outperformed in terms of accuracy, does but not holds good for large-scale database as the tree has to be traversed for a minimum time, based on the subset.

A feature based iris indexing algorithm for non-ideal poorquality iris image was proposed in [16]. In this approach segmentation was done at two stages, as the method is for nonideal image. The first step in above approach is to calculate approximately the inner and outer boundaries using elliptical model. The second step is to figure out the exact inner and outer boundaries of the iris using modified Mumford-shah functional [17]. The detected iris is then unwrapped into a rectangular region by converting into polar co-ordinates. To enhance the image the standard enhancement algorithm were used. The enhanced local regions are identified from each of the transformed images using a support vector machine based approach [18] and a single high quality image was generated. The textural feature and topological features [19] are extracted using Euler number. Later the textural and topological match scores are fused for further classification and indexing is performed. The indexing is done by a two-step process. The first step is used to generate a subset of possible match list and in the second step using $2 \mathrm{v}$-svm match score fusion was used, to find the best match from the possible matches. The result when compared with existing algorithm based on Euler code and $2 \mathrm{v}$-svm which proved to have better result in terms of accuracy which is $97.21 \%$ and time which the is less than $2 \mathrm{~s}$ approximately.

A new method for indexing iris images based on the context clustering property of the borrows-wheeler transform (BWT) was proposed [20]. The indexing scheme is based on the distribution of the patterns present in iris texture. The image acquisition and segmentation was based on the algorithm proposed by Shah \& Ross and normalization done based on the Daugman's rubber sheet model [11]. In next step, the normalized gray scale iris is converted into a binary image. A horizontal n-bit patter is chosen with the least value of coefficient of variation. The list is then sorted accordingly and a modified binary search algorithm is used. These patterns are found in the iris image and then the normalized iris image is divided into vertical segments based on the maximum occurrence of the n-bit patterns among these segments. An index value is assigned based on the segment number. The approach seems to work well with small scale databases.

Indexing based on hashing technique which do not sort iris template but on the other hand uses lower-dimensional hashes which are directly generated from the iris data, has also been proposed in [21]. In this technique the acquired image is preprocessed by discarding the top and bottom quarter say $315^{\circ}$ to $45^{\circ}$ and $135^{\circ}$ to $225^{\circ}$ of the iris, as they are often affected by eyelids and eyelashes. The preprocessed texture is used to extract a hash. Once the template is acquired a hash and a pointer is generated. The pointer generated is stored at the according node. If equal hashes were computed for different users, a linked list of pointer was stored at the according node. Thus the node can be reached by short paths. This technique addresses the problem of coarse classification and the hit rate is found to be better than other methods. The limitation in hashing technique is its scalability and it operates as a pure key generation and has no further user-specific secondary data.

\subsection{Based on Iris Color}

A novel indexing method based on natural iris color was proposed [22]. The input image is segmented based on Hough Circular transform to recognize the boundaries. The input image which was then converted from RGB space to $\mathrm{YCbCr}$ color space and partition is done based on the blue and red color indices. Depending on the intensity of red and blue color the bins are selected and stored into their respective bins. At the time of retrieval based on the color indices the bin is selected and the nearest identities are selected based on the set intersection between the bins. The system performs well for a small subset since there can be small intra-class variation which may lead to correct grouping. But for high degree of performance, the inter-class variation should be high ie database should be grouped with a large number of subsets, which again affects the search time and efficiency.

An indexing scheme which makes uses of iris color to determine an index and then use the index to reduce the search space in large iris databases was proposed in [23] The input is a color iris image, which is originally in RGB color space, is converted to chrominance of blue and red color $\mathrm{YCbCr}$. Since $\mathrm{YCbCr}$ color space allows the color 
indices to operate well in illumination a high indexing performance can be obtained. To identify an iris based on the color they had used color histogram was used, where each element contains the number of pixel having the color in the image and consider the pdf (probability density function) of the color value. Based on the color distribution, the image is distributed in different bins of the histogram and during matching its nearest neighbor was found by comparing all the bins in the database using K-NN algorithm. Using kd-tree identification and retrieval, the top best match from the subset was done. The system is robust and yields a good hit rate but the time required for a top best match is $\mathrm{O}(\log \mathrm{N}+\mathrm{k})$ where $\mathrm{k}$ is size of subset, and on average it needs to traverse at least one leaf node of the tree.

A novel iris indexing approach on noisy dataset and color as the indexing factor was proposed [24]. The noisy iris image is located for the region of interest (ROI) based on Adaboost method and the eyelids, eyelash spectaculars refection were removed from the image. After determining the ROI the iris color image was converted to RGB color space and classified accordingly. The average color value is normalized so as to keep away from bad consequence caused by illumination. The indexing is based on the color components with their internal length space interval between the color components. The complexity in this technique is that many of the coarse classification are not correctly classified and heterogeneous lighting condition influence the color distribution. Hence there is a need for color distribution computation which corrects the color based on standard calibration target method. The result seems to be more sensible that both of the normalized average values of each color component are close each other. The hit rate and penetration rate were computed before and after color correction and observably the HR after color correction is much higher than without color correction. The system after color calibration with small noisy dataset works well but when the size grows classification again can become a problem.

\subsection{Based on Iris Code}

A geometric hashing method to index a large iris biometric database has been proposed in [25], wherein the input iris image is preprocessed to produce noise independent image using Circular Hough transformation and a sector based approach. Later the local features were extracted by finding the keypoints in the image. These features are invariant to scaling and rotation and to some extent invariant to illumination, viewpoint and occlusion. The SFIT [26] is used for detecting keypoints descriptor from the preprocessed image. Further, orientation is approximated for each keypoints and the descriptor vector is defined relative to orientation. The indexing is then done based on detected keypoints. The keypoints are chosen for different combination of basis pair on the assumption that the other point should lie in positive axis. The hash table is formed based on the possible ordered basis pair where the keypoints are scaled to the magnitude of the keypoints equal to 1 . Done so, there are a number of entries in the hash table which leads to hash bin occupancy to a non-uniform entries with a peak of accumulated large number of entries. Hence an efficient technique for uniform distribution of entries in the hash table was proposed [27], by which rehashing function was applied to form uniform occupancy. For each entry found in the hash table bin a vote is casted and the votes greater than the threshold were considered to be potential matches and the top is considered as best match. A good accuracy of $98.5 \%$ has been obtained, as geometric hashing is inherently parallel where in the time required includes hashing and again for rehashing $29 \mathrm{~s}$ (approximate).

\section{PERFORMANCE METRICS AND IRIS DATABASES \\ EVALUATION}

The performance of the indexing technique and were generally evaluated based on penetration rate, hit rate, miss rate and redundancy rate.

- $\quad$ Penetration rate: The portion or fraction of the total database retrieved by the system for user identity.

- Hit rate: indicates the possibility that the correct identity belongs to at least one of the retrieved subset images.

- $\quad$ Redundancy rate: indicates the repetition frequency of the iris code when registering.

- $\quad$ Miss rate: indicates the possibility that the correct identity belongs to none of the retrieved type.

A brief overview of the various databases used for indexing are discussed and summarized in table 1.

- BATH database [28] is a publicly available database with a minimum of handling fees. It is maintained by university of Bath in conjunction with Smart Sensor Limited. The databases consist of 1600 eyes taken from 800 people with 20 images of each left and right eye. The iris images are with higher resolution of 1280x960 pixel stored in JPEG format.

- ICE (Iris Challenge Evaluation) [29] a large database used for evaluation of iris recognition system. It is conducted and managed by National Institute of Standards and Technology (NIST). The database consist of 2953 iris images form 132 subjects. The iris images are $480 \times 640$ pixel resolution. The images are acquired under near infrared (NIR) in a constrained environment. The images are stored in tif file format. The main objective is to identify the correlation between the left and right irises for match and non-match similarity scores.

- UBIRIS [30] is a public and free noisy iris database managed by the Dept of CS at University of Beira Interior. The database consist of 1877 iris images collected from 372 subjects, 5 samples each in two sessions. During the first session, images are captured under a controlled environment to minimize the noise factor. In the second session images were acquired form 131 persons in a natural luminosity location. The images are stored in JPEG file format.

- IITK Indian Institute of Technology, Kanpur iris image database is used for the iris data. The database comprises of 1350 iris images of 150 subjects (nine images per subject) from their left eye. The images are JPEG in grey scale and are resized with $450 \times 350$ pixels resolution.

- CASIA [31] Iris Image Database for Testing Version 1.0 (or IR-TestV1) contains 10,000 iris images of 2,000 eyes from 1,000 subjects. The images are 8 bit gray-scale BMP files with a resolution of $640 x 480$. CASIA Iris Image Database Version 1.0 (CASIA-IrisV1) includes 756 iris images from 108 eyes. For each eye, 7 images are captured in two sessions where three samples are collected in the first session and four in the second session. All images are stored as BMP format with resolution 320x280. CASIA Iris Image Database Version 2.0 (CASIA-IrisV2) includes two subsets captured with two different devices in a controlled environment. Each subset includes 1200 images from 60 classes. 
CASIA-IrisV3 includes three subsets which are labeled as CASIA-Iris-Interval, CASIA-Iris-Lamp, CASIA-Iris-Twins. CASIA-IrisV3 contains a total of 22,034 iris images from more than 700 subjects. All iris images are 8 bit gray-level JPEG files, collected under near infrared illumination. Almost all subjects are Chinese except a few in CASIA-IrisInterval. Because the three data sets were collected in different times, only CASIA-Iris-Interval and CASIA-Iris-
Lamp have a small overlap in subjects. CASIA-Iris-Twins contains iris images of 100 pairs of twins, which were collected during Annual Twins Festival in Beijing. Although iris is usually regarded as a kind of phenotypic biometric characteristics and even twins have their unique iris patterns, it is interesting to study the dissimilarity and similarity between iris images of twins.

Fig 1: Various Iris Databases

\begin{tabular}{|c|c|c|c|c|}
\hline Database & Size (no of subject) & Resolution & File Format & Remarks \\
\hline BATH & $\begin{array}{l}1600 \text { eyes from } 800 \\
\text { subjects }\end{array}$ & $1280 \times 960$ & JPEG & $\begin{array}{l}\text { - } \\
\text { - }\end{array}$ \\
\hline ICE & $\begin{array}{l}2953 \text { iris from } 132 \\
\text { subjects }\end{array}$ & $480 \times 640$ & TIF & $\begin{array}{l}\text { - Used for evaluation } \\
\text { left and right irises }\end{array}$ \\
\hline UBIRIS & $\begin{array}{l}1877 \text { iris from } 372 \\
\text { subjects }\end{array}$ & - & JPEG & Noisy database \\
\hline CASIA & $\begin{array}{l}22,034 \text { iris from more } \\
\text { than } 700 \text { subjects }\end{array}$ & $320 \times 280$ & JPEG & $\begin{array}{ll} & \text { Have four versions } \\
\text { - } & \text { Larger dataset and used for research }\end{array}$ \\
\hline IITK & $\begin{array}{l}1350 \text { iris of } 150 \\
\text { subjects from left eye }\end{array}$ & $450 \times 350$ & JPEG & Noisy database \\
\hline
\end{tabular}

\section{CONCLUDING REMARKS}

From the survey carried out on the indexing methodology based on the iris feature, it is seen that the various technique are mainly based on tree traversal. However, they differ in the way the tree is built and traversed. The tree traversing techniques used are kd-tree, B-tree. Apart from these there are some more like: $\mathrm{R}$-tree, $\mathrm{R}+$ tree, $\mathrm{B}$ +tree where all these technique suffers from "curse of dimensionality". The other indexing method is hashing, which suffers from pure key generation. The size of data bases used is small, when compare to actual field data for which indexing techniques have to be developed, especially for a large database like UID in India. Further, indexing using noisy iris data is rather rare, but is likely to be expected in a real-life project. Further research to address the above issues are the need of the hour and this survey has shown the research potential available in the years to come, in the area of iris indexing for large databases with noise.

\section{REFERENCES}

[1] Jain, A. Ross and S. Prabhakar, "An Introduction to Biometric Recognition," IEEE Transactions on Circuits and Systems on Video Technology, vol. 14, no. 1, pp. 420, Jan 2004.

[2] K. W. Bowyer, K. Hollingsworth, and P. J. Flynn, "Image understanding for iris biometrics: A survey," Computer. Vis. Image Understand., vol. 110, no. 2, pp. 281-307, May 2008.

[3] U. I. D. of India. http://www.uidai.gov.in/, Last accessed on Feb 02, 2012.
[4] L. Ma, T. Tan, Y. Wang, and D. Zhang, "Efficient iris recognition by characterizing key local variations," IEEE Trans. Image Process., vol. 13, no. 6, pp. 739-750, Jun. 2004.

[5] R. Mukherjee and A. Ross, "Indexing iris images," in Proc. 19th Int.Conf. Pattern Recognit., 2008, pp. 1-4.

[6] Yulin Si, Jiangyuan Mei, and Huijun Gao, "Novel Approaches to Improve Robustness, Accuracy and Rapidity of Iris Recognition Systems", IEEE Transactions on Industrial Informatics, vol. 8, no. 1, pp.110-117, February 2012.

[7] P. Hsiao, C. Lu, and L. Fu, "Multilayered image processing for multiscale Harris corner detection in digital realization," IEEE Trans. Ind.Electron., vol. 57, no. 5, pp. 1799-1805, 2010.

[8] H. Mehrotra, B. Majhi, and P. Gupta, "Robust iris indexing scheme using geometric hashing of SIFT keypoints,” J. Network Comput. Appl., vol. 33, no. 3, pp. 300-313, 2010.

[9] Hunny Mehrotra, Badrinath G. Srinivas, Banshidhar Majhi,and Phalguni Gupta, "Indexing Iris Biometric Database Using Energy Histogram of DCT Subbands", Journal of Communication in computer and Information Science, vol. 40, pp. 194-204, 2009.

[10] D.J.Kerbyson, T.J.Atherton, "Circle detection using Hough transform filters" Fifth International Conference on Image Processing and its Applications, pp. 370-374, 1995.

[11] John Daugman, "How Iris Recognition Works", IEEE Transactions on Circuits And Systems For Video Technology, Vol. 14, No.1, January 2004. 
[12] S.A.Khayam, "The Discrete Cosine Transform (DCT): Theory and Application", Tutorial Report, Michigan State University, 2003.

[13] U.Jayaraman, S.Prakash, and P.Gupta, "An efficient technique for indexing multimodal biometric databases", International. Journal of Biometrics, vol. 1, no. 4, pp.418-441, 2009.

[14] L.Ma, T.N.Tan, Y.H.Wang, and D.Zhang, "Local intensity variation analysis for iris Recognition", Pattern Recognition, Vol. 37, No. 6, pp.1287-1298, 2004.

[15] R.C.Gonzalez, and R.E.Woods, "Digital image processing", Addison-Wesley Longman Publishing Co., Inc. 2001.

[16] Mayank Vatsa, Richa Singh, Afzel Noore, "Improving Iris Recognition Performance Using Segmentation, Quality Enhancement, Match Score Fusion, and Indexing" IEEE Transactions on Systems, Man, and Cybernetics, Part B vol. 38, no.4, pp. 1021-1035, 2008

[17] Tsai, A. Yezzi, Jr., and A.Willsky, "Curve evolution implementation of the Mumford-Shah functional for image segmentation, denoising, interpolation, and magnification," IEEE Trans. Image Process., vol. 10, no. 8, pp. 1169-1186, Aug. 2001.

[18] R. Singh, M. Vatsa, and A. Noore, "Improving verification accuracy by synthesis of locally enhanced biometric images and deformable model," Signal Process., vol. 87, no. 11, pp. 2746-2764, Nov. 2007.

[19] M. Vatsa, R. Singh, and A. Noore, "Reducing the false rejection rate of iris recognition using textural and topological features," Int. J. SignalProcess., vol. 2, no. 1, pp. 66-72, 2005

[20] Ravindra Gadde, D.Adjeroh, A.Ross, "Indexing Iris Images Using Burrow Wheelers Transform", Proc of IEEE International Workshop on Information Forensics and Security, Dec 2010.
[21] C. Rathgeb, A. Uhl, "Iris-Biometric Hash Generation for Biometric Database Indexing", 20th International. Conference on Pattern Recognition, Dec 2010.

[22] N. B. Puhan, N. Sudha, "A novel iris database indexing method using the iris color," Proc. 3rd IEEE Conf. on Industrial Electronics and Applications (ICIEA), pp. 1886-1891, 2008

[23] Umarani J, Surya Prakash and Phalguni Gupta, "An Iris Retrieval Technique Based on Color and Texture", Indian Conference on Computer Vision, Graphics and Image Processing, pp. 93-100, Dec 2010.

[24] Qin Zhao, "A new approach for noisy iris database indexing based on color information", The 6th International Conference on Computer Science \& Education, pp 28-31, August 2011.

[25] Hunny Mehrotra, Banshidhar Majhi, Phalguni Gupta, "Robust iris indexing scheme using geometric hashing of SIFT keypoints", Journal of Network and Computer Applications vol.33 no.3, pp. 300-313, 2010.

[26] D.G. Lowe, "Distinctive image features from scaleinvariant keypoints", International Journal on Computer Vision, vol. 60, no.2, pp.91-110, 2004.

[27] Rigoutsos, R. Hummel, "Implementation of geometric hashing on the Connection Machine" Workshop on directions in automated CAD-based vision, pp. 76-84, 1991.

[28] Michal Dobeš and Libor Machala, Iris Database, http://www.inf.upol.cz/iris/

[29] [Online]. http://iris.nist.gov/ice/ICE Home.htm

[30] H. Proenca and Alexandre, "UBIRIS: A Noisy Iris Image Database", Lecture Notes in Computer Science, vol. 1 pp. 970-977, 2005.

[31] Online.Available:http://www.cbsr.ia.ac.cn/IrisDatabase/ir isdatabase.php 